\title{
Joint monotone and boolean numerical and spectral radii of $d$-tuples of operators
}

\section{Anna Kula ${ }^{1} \cdot$ Janusz Wysoczański ${ }^{1}$}

Received: 4 February 2020 / Accepted: 16 April 2020 / Published online: 2 June 2020

(C) The Author(s) 2020

\begin{abstract}
We study joint numerical and spectral radii defined for $d$-tuples of bounded operators on a Hilbert space and related to noncommutative notions of independence. The definitions are in analogy with the ones of Popescu, where his formulations turned out to be related with free creation operators, and in this way related to the free independence of Voiculescu. In our study the definitions are related with either weakly monotone creation operators, and thus associated with the monotone independence of Muraki, or with boolean creation operators, and hence related with the boolean independence.
\end{abstract}

Keywords Numerical radius $\cdot$ Spectral radius $\cdot$ Fock space $\cdot$ Monotone independence $\cdot$ Boolean independence

Mathematics Subject Classification 46L53 - 47A12 - 47A13

\section{Introduction}

The notion of numerical radius as well as the related notion of numerical range is an object of intensive studies since the work by Toeplitz [10] in 1918 until today. Numerical radius provides a norm on the space of bounded operators, which is equivalent to the operator norm. Its special features include unitarity invariance, the

Communicated by Lyudmila Turowska.

Dedicated to Franciszek Hugon Szafraniec on the occasion of his 80th birthday, with appreciation of his influence on our multidimensional collaboration.

Janusz Wysoczański

janusz.wysoczanski@math.uni.wroc.pl

Anna Kula

anna.kula@math.uni.wroc.pl

1 Institute of Mathematics, University of Wrocław, Wrocław, Poland 
power inequality and the relation with the spectral radius, see e.g. [6]. The numerical range and radius link the properties of operators with geometry of a complex plane, allowing many interesting applications, from the approximate localization of spectrum via the stability results for differential equations (e.g. [5]) to the von Neumann type inequalities (e.g. [1]).

In 2009, Gelu Popescu [9], in his search for a free analogue of the Sz.-NagyFoias theory for row contractions, defined free analogues of numerical and spectral radii, namely the joint numerical radius and joint spectral radius for $d$-tuples of operators $\left(T_{1}, \ldots, T_{d}\right)$ acting on a Hilbert space $\mathcal{H}$. The definitions are as follows.

Definition 1 The (free) joint numerical radius is

$$
w_{F}\left(T_{1}, \ldots, T_{d}\right):=\sup \left\{\left|\sum_{\alpha \in \mathbb{F}_{d}^{+}} \sum_{j=1}^{d}\left\langle T_{j} h_{g_{j} \alpha} \mid h_{\alpha}\right\rangle\right|: \sum_{\alpha \in \mathbb{F}_{d}^{+}}\left\|h_{\alpha}\right\|^{2}<\infty\right\}
$$

where $\mathbb{F}_{d}^{+}$is the free semigroup with free generators $g_{1}, \ldots, g_{d}$ and each $\alpha \in \mathbb{F}_{d}^{+}$is a word in these generators.

Definition 2 The (free) joint spectral radius is

$$
r_{F}\left(T_{1}, \ldots, T_{d}\right):=\lim _{k \rightarrow \infty}\left\|\sum_{|\alpha|=k} T_{\alpha} T_{\alpha}^{*}\right\|^{1 / 2 k}
$$

where for $\alpha \in \mathbb{F}_{d}^{+}$one puts $|\alpha|=k$ if it is a word in $k$ generators $\alpha=g_{i_{1}} \ldots g_{i_{k}}$.

These two notions are related not only to the free semigroup $\mathbb{F}_{d}^{+}$, but also to the model of freeness of Voiculescu [11], and more precisely to the creation operators on the full Fock space by the following result.

Theorem 3 ([9], Corollary 1.2) The joint free numerical and spectral radii can be computed as the ordinary numerical radius $w$ and the spectral radius $r$ of single operators:

$$
\begin{gathered}
w_{F}\left(T_{1}, \ldots, T_{d}\right)=w\left(S_{1} \otimes T_{1}^{*}+\ldots+S_{d} \otimes T_{d}^{*}\right) \\
r_{F}\left(T_{1}, \ldots, T_{d}\right)=r\left(S_{1} \otimes T_{1}^{*}+\ldots+S_{d} \otimes T_{d}^{*}\right),
\end{gathered}
$$

where $S_{1}:=S\left(e_{1}\right), \ldots, S_{d}:=S\left(e_{d}\right)$ are the free creation operators on the full Fock space $\mathcal{F}\left(H_{d}\right)$ on d-dimensional Hilbert space $H_{d}$ with an orthonormal basis $\left\{e_{1}, \ldots, e_{d}\right\}$ and $S(h)$ is the creation operator by the vector $h \in H_{d}: S(h) v=h \otimes v$ for $v \in \mathcal{F}\left(H_{d}\right)$.

The main idea of this paper is to study analogues of these definitions in the case where we replace the (free) full Fock space by a Fock space associated to other noncommutative independences, and the free creation operators by the creation operators on the appropriate Fock space. We show that the joint (noncommutative) numerical radii, defined in analogy to Theorem 1 , satisfy many basic properties similar to the free case. In particular, we show the unitarity invariance and the relation with the 
appropriately defined spectral radius. We also compute some examples. In the paper we treat the monotone and boolean case, but the framework is more general. Our idea establishes yet another bridge between classical operator theory and the noncommutative probability and we believe this is a starting point for further investigations. However, in this paper there is no need to define the monotone and boolean independences, it is sufficient to consider the models of both of them, built on either weakly monotone Fock space or on the Boolean Fock space, respectively.

\section{General scheme}

Recall that the (classical) numerical radius of a linear operator $T$, bounded on a Hilbert space $\mathcal{H}$, is defined by

$$
w_{\mathrm{cl}}(T):=\sup \{|\langle T h, h\rangle|: h \in \mathcal{H},\|h\|=1\}
$$

and the (classical) spectral radius of $T$ is defined by

$$
r_{\mathrm{cl}}(T)=\lim _{m \rightarrow \infty}\left\|T^{m}\right\|^{\frac{1}{m}}
$$

Let us consider one of the noncommutative independence, e.g. boolean, free, monotone and let us consider a noncommutative Fock space $F_{\star}(H)$ associated to this independence. By this we mean the full Fock space ( [11]) for the free independence of Voiculescu, the weakly monotone Fock space ( [12]) for the monotone independence of Muraki and the boolean Fock space ( [3]) for the boolean independence (c.f. [2]). On each of these Fock spaces we are given creation and annihilation operators, which we shall use to define relative joint (numerical and spectral) radii, following the work by Popescu [9] for the free case.

Let $d \in \mathbb{N}$ and let $H$ be the $d$-dimensional Hilbert space with the orthonormal basis $\left\{e_{j} ; 1 \leq j \leq d\right\}$. Denote by $A_{1}^{\star}, \ldots, A_{d}^{\star}$ the creation operators associated to the basic vectors $A_{j}^{\star}=A^{\star}\left(e_{j}\right)$ on the related Fock space $F_{\star}(H)$ and define the $\star$-joint numerical radius of the $d$-tuple $\left(T_{1}, \ldots, T_{d}\right)$ of operators in $B(\mathcal{H})$ by

$$
w_{\star}\left(T_{1}, \ldots, T_{d}\right):=w_{\mathrm{cl}}\left(A_{1}^{\star} \otimes T_{1}^{*}+\ldots A_{d}^{\star} \otimes T_{d}^{*}\right) .
$$

Similarly, we define the $\star$-joint spectral radius of $\left(T_{1}, \ldots, T_{d}\right)$ by

$$
r_{\star}\left(T_{1}, \ldots, T_{d}\right):=r_{\mathrm{cl}}\left(A_{1}^{\star} \otimes T_{1}^{*}+\ldots A_{d}^{\star} \otimes T_{d}^{*}\right) .
$$

The following properties of the $\star$-joint numerical and spectral radii are immediate consequences of the construction and of the properties of the classical numerical radius (compare with Theorem 1.1 in [9]).

Proposition 4 The $\star$-joint numerical radius and joint spectral radius satisfy:

(i) $\quad w_{\star}\left(\lambda T_{1}, \ldots, \lambda T_{d}\right)=|\lambda| w_{\star}\left(T_{1}, \ldots, T_{d}\right)$ for any $\lambda \in \mathbb{C}$;

(ii) $w_{\star}\left(T_{1}+T_{1}^{\prime}, \ldots, T_{d}+T_{d}^{\prime}\right) \leq w_{\star}\left(T_{1}, \ldots, T_{d}\right)+w_{\star}\left(T_{1}^{\prime}, \ldots, T_{d}^{\prime}\right)$;

(iii) $w_{\star}\left(U^{*} T_{1} U, \ldots, U^{*} T_{d} U\right)=w_{\star}\left(T_{1}, \ldots, T_{d}\right)$ for any unitary $U: \mathcal{K} \rightarrow \mathcal{H}$; 
(iv) $\quad w_{\star}\left(I_{\mathcal{K}} \otimes T_{1}, \ldots, I_{\mathcal{K}} \otimes T_{d}\right)=w_{\star}\left(T_{1}, \ldots, T_{d}\right)$ for any separable Hilbert space $\mathcal{K}$

(v) $\quad r_{\star}\left(T_{1}, \ldots, T_{d}\right) \leq w_{\star}\left(T_{1}, \ldots, T_{d}\right)$.

Proof The properties (i) and (ii) follows from $w_{\mathrm{cl}}(\lambda T)=|\lambda| w_{\mathrm{cl}}(T)$ and $w_{\mathrm{cl}}\left(T+T^{\prime}\right) \leq w_{\mathrm{cl}}(T)+w_{\mathrm{cl}}\left(T^{\prime}\right)$. As for (iii), for a unitary $U: \mathcal{K} \rightarrow \mathcal{H}$ we set $V=$ $I \otimes U$ and $A:=A_{1}^{\star} \otimes T_{1}^{*}+\ldots A_{d}^{\star} \otimes T_{d}^{*}$ and observe that

$$
\begin{aligned}
w_{\star} & \left(U^{*} T_{1} U, \ldots, U^{*} T_{d} U\right)=w_{\mathrm{cl}}\left(A_{1}^{\star} \otimes U^{*} T_{1}^{*} U+\ldots A_{d}^{\star} \otimes U^{*} T_{d}^{*} U\right) \\
& =w_{\mathrm{cl}}\left((I \otimes U)^{*}\left(A_{1}^{\star} \otimes T_{1}^{*}+\ldots A_{d}^{\star} \otimes T_{d}^{*}\right)(I \otimes U)\right)=w_{\mathrm{cl}}\left(V^{*} A V\right) \\
& =\sup \{|\langle A V h, V h\rangle|: h \in \mathcal{H},\|h\|=1\} \\
& =\sup \left\{\left|\left\langle A h^{\prime}, h^{\prime}\right\rangle\right|: h^{\prime}=V h \in \mathcal{F}_{*}(H) \otimes \mathcal{K},\left\|h^{\prime}\right\|=\|V h\|=1\right\} \\
& =w_{\mathrm{cl}}(A)=w_{\star}\left(T_{1}, \ldots, T_{d}\right) .
\end{aligned}
$$

The property $(i v)$ goes exactly as in the proof of [9, Theorem 1.1], using $w_{\mathrm{cl}}\left(I_{\mathcal{K}} \otimes T\right)=w_{\mathrm{cl}}(T)$ :

$$
\begin{aligned}
w_{\star}\left(I_{\mathcal{K}} \otimes T_{1}, \ldots, I_{\mathcal{K}} \otimes T_{d}\right) & =w_{\mathrm{cl}}\left(\sum_{j=1}^{d} A_{j}^{\star} \otimes\left(I_{\mathcal{K}} \otimes T_{j}\right)\right) \\
& =w_{\mathrm{cl}}\left(I_{\mathcal{K}} \otimes\left(\sum_{j=1}^{d} A_{j}^{\star} \otimes T_{j}\right)\right)=w_{\mathrm{cl}}\left(\sum_{j=1}^{d} A_{j}^{\star} \otimes T_{j}\right) \\
& =w_{\star}\left(T_{1}, \ldots, T_{d}\right) .
\end{aligned}
$$

Finally, to show $(v)$ we just use the classical result $r_{\mathrm{cl}}(T) \leq w_{\mathrm{cl}}(T)$.

\section{Joint boolean numerical and spectral radii}

Recall after [3] that the boolean Fock space (over the $d$-dimensional space $H$ ) is defined as the direct sum

$$
\mathcal{F}_{B}(H)=\mathbb{C} \Omega \oplus H,
$$

where $\Omega$ is a unit vector, called the vacuum. The boolean creation and annihilation operators are given by

$$
B^{*}(f) h=\left\{\begin{array}{ll}
f & \text { if } h=\Omega \\
0 & \text { if } h \in H
\end{array}, \quad B(f) h=\left\{\begin{array}{cl}
0 & \text { if } h=\Omega \\
\langle f, h\rangle \Omega & \text { if } h \in H
\end{array} .\right.\right.
$$

For a fixed orthonormal basis $\left\{e_{j}: 1 \leq j \leq d\right\}$ in $H$, we shall use the notation $B_{j}^{*}:=B^{*}\left(e_{j}\right), B_{j}:=B\left(e_{j}\right), j=1, \ldots, d$, for the creation and annihilation operators (respectively) by the basic vectors, and $e_{0}:=\Omega$. It is easy to see that $B_{j} B_{k}^{*}=\delta_{j k} P_{\Omega}$, where $P_{\Omega}$ is the projection onto the vacuum vector $e_{0}=\Omega$.

Let now $\left(T_{1}, \ldots, T_{d}\right)$ be the $d$-tuple of bounded operators on a Hilbert space $\mathcal{H}$. We define the joint boolean numerical radius of $\left(T_{1}, \ldots, T_{d}\right)$ as 


$$
w_{B}\left(T_{1}, \ldots, T_{d}\right):=w_{\mathrm{cl}}\left(B_{1}^{*} \otimes T_{1}^{*}+\ldots B_{d}^{*} \otimes T_{d}^{*}\right)
$$

and the spectral joint boolean spectral radius of $\left(T_{1}, \ldots, T_{d}\right)$ as

$$
r_{B}\left(T_{1}, \ldots, T_{d}\right):=r_{\mathrm{cl}}\left(B_{1}^{*} \otimes T_{1}^{*}+\ldots B_{d}^{*} \otimes T_{d}^{*}\right) .
$$

We first provide the explicit formula for computing the joint boolean numerical radius, which is the analogue of Popescu's definition in the free case (see Definition 1).

Proposition 5 The joint boolean numerical radius can be expressed as

$$
w_{B}\left(T_{1}, \ldots, T_{d}\right)=\sup \left\{\left|\left\langle g_{0}, \sum_{j=1}^{d} T_{j} g_{j}\right\rangle\right|: g_{0}, g_{1}, \ldots, g_{d} \in \mathcal{H}, \sum_{j=0}^{d}\left\|g_{j}\right\|^{2}=1\right\} .
$$

Proof By the definition of the classical numerical radius, we have

$$
w_{B}\left(T_{1}, \ldots, T_{d}\right):=\sup \left\{\left|\left\langle\sum_{j=1}^{d} B_{j}^{*} \otimes T_{j}^{*} h, h\right\rangle\right|: h \in \mathcal{F}_{B}(H) \otimes \mathcal{H},\|h\|=1\right\} .
$$

Expressing $h \in \mathcal{F}_{B}(H) \otimes \mathcal{H}$ as $h=\sum_{k=0}^{d} e_{k} \otimes g_{k}$ with $g_{0}, g_{1}, \ldots, g_{d} \in \mathcal{H}$ (recall that $e_{0}:=\Omega$ ), we observe that

$$
\|h\|^{2}=\left\langle\sum_{k=0}^{d} e_{k} \otimes g_{k}, \sum_{m=0}^{d} e_{m} \otimes g_{m}\right\rangle=\sum_{k, m=0}^{d}\left\langle e_{k}, e_{m}\right\rangle\left\langle g_{k}, g_{m}\right\rangle=\sum_{k=0}^{d}\left\|g_{k}\right\|^{2},
$$

whereas, using the relation $B_{j} e_{m}=\delta_{j m} e_{0}$, for $1 \leq j, m \leq d$, and $B_{j} e_{0}=0$ for $1 \leq j \leq d$, we get

$$
\begin{aligned}
\left\langle\sum_{j=1}^{d} B_{j}^{*} \otimes T_{j}^{*} h, h\right\rangle & =\sum_{j=1}^{d} \sum_{k, m=0}^{d}\left\langle\left(B_{j}^{*} \otimes T_{j}^{*}\right)\left(e_{k} \otimes g_{k}\right),\left(e_{m} \otimes g_{m}\right)\right\rangle \\
& =\sum_{j=1}^{d} \sum_{k, m=0}^{d}\left\langle B_{j}^{*} e_{k}, e_{m}\right\rangle\left\langle T_{j}^{*} g_{k}, g_{m}\right\rangle \\
& =\sum_{j=1}^{d} \sum_{k, m=0}^{d}\left\langle e_{k}, B_{j} e_{m}\right\rangle\left\langle T_{j}^{*} g_{k}, g_{m}\right\rangle \\
& =\sum_{m=1}^{d} \sum_{k=0}^{d}\left\langle e_{k}, e_{0}\right\rangle\left\langle g_{k}, T_{m} g_{m}\right\rangle \\
& =\sum_{m=1}^{d}\left\langle g_{0}, T_{m} g_{m}\right\rangle=\left\langle g_{0}, \sum_{m=1}^{d} T_{m} g_{m}\right\rangle .
\end{aligned}
$$


It turns out that all of the properties that were shown to hold for the (free) joint numerical radius (see [9, Theorem 1.1]), remains true in the boolean case. Some of them were already observed in Proposition 4; here we prove the remaining ones.

Proposition 6 We have

$(i)_{B} \quad w_{B}\left(T_{1}, \ldots, T_{d}\right)=0$ if and only if $T_{1}=\ldots=T_{d}=0$,

$(\text { ii })_{B} \quad \frac{1}{2}\left\|\sum_{j=1}^{d} T_{j} T_{j}^{*}\right\|^{\frac{1}{2}} \leq w_{B}\left(T_{1}, \ldots, T_{d}\right) \leq\left\|\sum_{j=1}^{d} T_{j} T_{j}^{*}\right\|^{\frac{1}{2}}$,

$(\text { iii })_{B} \quad w_{B}\left(X^{*} T_{1} X, \ldots, X^{*} T_{d} X\right) \leq\|X\|^{2} w_{B}\left(T_{1}, \ldots, T_{d}\right)$ for any bounded operator $X: \mathcal{H} \rightarrow \mathcal{K}$.

Proof $\operatorname{Ad}(i)_{B}$. By the properties of the classical numerical radius, $w_{B}\left(T_{1}, \ldots, T_{d}\right)=$ 0 implies $\sum_{j=1}^{d} B_{j}^{*} \otimes T_{j}^{*}=0$. Hence for any $h=\sum_{k=0}^{d} e_{k} \otimes g_{k} \in \mathcal{F}_{B}(H) \otimes \mathcal{H}$, one gets

$$
0=\sum_{j=1}^{d} B_{j}^{*} \otimes T_{j}^{*} h=\sum_{j=1}^{d} \sum_{k=0}^{d} B_{j}^{*} e_{k} \otimes T_{j}^{*} g_{k}=\sum_{j=1}^{d} B_{j}^{*} e_{0} \otimes T_{j}^{*} g_{0}=\sum_{j=1}^{d} e_{j} \otimes T_{j}^{*} g_{0} .
$$

Thus, for any $j=1, \ldots, d$ and $g_{0} \in \mathcal{H}$, one gets $T_{j}^{*} g_{0}=0$. Consequently, $T_{j}^{*}=0$ and, equivalently, $T_{j}=0$ for any $j=1, \ldots, d$.

Ad $(i i)_{B}$. Thanks to $\frac{1}{2}\|T\| \leq w_{c l}(T) \leq\|T\|$, we have

$$
\begin{aligned}
w_{B}\left(T_{1}, \ldots, T_{d}\right) & =w_{c l}\left(\sum_{j=1}^{d} B_{j}^{*} \otimes T_{j}^{*}\right) \leq\left\|\sum_{j=1}^{d} B_{j}^{*} \otimes T_{j}^{*}\right\| \\
& =\left\|\left(\sum_{j=1}^{d} B_{j}^{*} \otimes T_{j}^{*}\right)^{*}\left(\sum_{k=1}^{d} B_{k}^{*} \otimes T_{k}^{*}\right)\right\|=\|\sum_{j, k=1}^{d} \underbrace{B_{j}^{*} B_{k}}_{=\delta_{j k} P_{\Omega}} \otimes T_{j}^{*} T_{k}\|^{\frac{1}{2}} \| \\
& =\left\|P_{\Omega} \otimes \sum_{j=1}^{d} T_{j}^{*} T_{j}\right\|^{\frac{1}{2}}=\underbrace{\left\|P_{\Omega}\right\|^{\frac{1}{2}}}_{=1} \cdot\left\|\sum_{j=1}^{d} T_{j}^{*} T_{j}\right\|^{\frac{1}{2}} .
\end{aligned}
$$

Similarly, we prove that $w_{B}\left(T_{1}, \ldots, T_{d}\right) \geq \frac{1}{2}\left\|\sum_{j=1}^{d} T_{j}^{*} T_{j}\right\|^{\frac{1}{2}}$.

Ad $(i i i)_{B}$. Let $X: \mathcal{H} \rightarrow \mathcal{K}$ be a bounded operator and let $g_{0}, \ldots, g_{d} \in \mathcal{H}$ satisfy $\sum_{k=0}^{d}\left\|g_{k}\right\|^{2}=1$. Define $C:=\sqrt{\sum_{k=0}^{d}\left\|X g_{k}\right\|^{2}}$ and $h_{k}:=\frac{1}{C} X g_{k}, k=0,1, \ldots, d$. Then $\sum_{k=0}^{d}\left\|h_{k}\right\|^{2}=1$ and $C \leq\|X\|$. Consequently, 


$$
\begin{aligned}
w_{B}\left(X^{*} T_{1} X, \ldots, X^{*} T_{d} X\right) & =\sup \left\{\left|\left\langle X g_{0}, \sum_{j=1}^{d} T_{j} X g_{j}\right\rangle\right|: \sum_{j=0}^{d}\left\|g_{j}\right\|^{2}=1\right\} \\
& \leq \sup \left\{\left|\left\langle C h_{0}, \sum_{j=1}^{d} T_{j} C h_{j}\right\rangle\right|: \sum_{j=0}^{d}\left\|h_{j}\right\|^{2}=1\right\} \\
& =C^{2} w_{B}\left(T_{1}, \ldots, T_{d}\right) \leq\|X\|^{2} w_{B}\left(T_{1}, \ldots, T_{d}\right) .
\end{aligned}
$$

Remark 7 The properties $(i)$, $(i i)$ and $(i)_{B}$ show that the joint boolean numerical radius is a norm on $B(\mathcal{H})^{d}$, which, by $(i i)_{B}$, is actually equivalent to the operator norm $\left\|\sum_{j=1}^{d} T_{j}^{*} T_{j}\right\|^{\frac{1}{2}}$ of the operator row matrix $\left[T_{1}, \ldots, T_{d}\right]$, hence $w_{B}$ is a continuous map in the norm topology.

We now compute some examples and, in particular, show that $w_{B} \neq w_{F}$.

Example 8 For $T_{j}=I_{\mathcal{H}}, j=1, \ldots, d$ we have

$$
w_{B}\left(I_{1}, \ldots, I_{d}\right)=\frac{\sqrt{d}}{2} .
$$

Using the relation between the $\ell_{1}$ - and $\ell_{2}$-norm on $\mathcal{H}^{d}$ :

$$
\sum_{k=1}^{d}\left\|g_{k}\right\| \leq \sqrt{d} \sqrt{\sum_{k=1}^{d}\left\|g_{k}\right\|^{2}} \quad \text { for } g_{1}, \ldots, g_{d} \in \mathcal{H}
$$

and Proposition 5, we observe that

$$
\left|\left\langle g_{0}, \sum_{k=1}^{d} g_{k}\right\rangle\right| \leq\left\|g_{0}\right\| \cdot\left\|\sum_{k=1}^{d} g_{k}\right\| \leq\left\|g_{0}\right\| \cdot \sum_{k=1}^{d}\left\|g_{k}\right\| \leq \sqrt{d}\left\|g_{0}\right\| \cdot \sqrt{\sum_{k=1}^{d}\left\|g_{k}\right\|^{2}} .
$$

Hence for any $g_{0}, \ldots, g_{d} \in \mathcal{H} \quad$ such that $\sum_{k=0}^{d}\left\|g_{k}\right\|^{2}=1$, denoting $t:=\left\|g_{0}\right\| \in[0,1]$, we get

$$
\begin{aligned}
w_{B}\left(I_{1}, \ldots, I_{d}\right) & \leq \sup \left\{\sqrt{d}\left\|g_{0}\right\| \cdot \sqrt{\sum_{k=1}^{d}\left\|g_{k}\right\|^{2}}: g_{0}, \ldots, g_{d} \in \mathcal{H}, \sum_{k=0}^{d}\left\|g_{k}\right\|^{2}=1\right\} \\
& =\sup \left\{t \cdot \sqrt{d} \cdot \sqrt{1-t^{2}}: t \in[0,1]\right\}=\frac{\sqrt{d}}{2},
\end{aligned}
$$

and the supremum is achieved when $t=\frac{1}{\sqrt{2}}$. This show that $w_{B}\left(I_{1}, \ldots, I_{d}\right) \leq \frac{\sqrt{d}}{2}$.

To see that the equality holds, take $g_{0} \in \mathcal{H}$ with $\left\|g_{0}\right\|^{2}=\frac{1}{2}$ and $g_{k}=\frac{g_{0}}{\sqrt{d}}$, $k=1, \ldots, d$. Then $\sum_{k=1}^{d}\left\|g_{k}\right\|^{2}=1$ whereas $\left|\left\langle g_{0}, \sum_{k=1}^{d} g_{k}\right\rangle\right|=\left|\left\langle g_{0}, \frac{d}{\sqrt{d}} g_{0}\right\rangle\right|=\frac{\sqrt{d}}{2}$. 
Example 9 For $T_{j}=B_{j}, j=1, \ldots, d$, the boolean annihilators, we have

$$
w_{B}\left(B_{1}, \ldots, B_{d}\right)=\frac{\sqrt{d}}{2} .
$$

Since $B_{j} h=\left\langle h, e_{j}\right\rangle \Omega$ for $h \in \mathcal{H}$, we compute

$$
\begin{aligned}
\left|\left\langle g_{0}, \sum_{j=1}^{d} B_{j} g_{j}\right\rangle\right| & =\left|\left\langle g_{0}, \sum_{j=1}^{d}\left\langle g_{j}, e_{j}\right\rangle \Omega\right\rangle\right| \leq\left\|g_{0}\right\|\left|\sum_{j=1}^{d}\left\langle g_{j}, e_{j}\right\rangle\right| \\
& \leq\left\|g_{0}\right\| \sum_{j=1}^{d}\left\|g_{j}\right\| \leq \sqrt{d}\left\|g_{0}\right\| \sqrt{\sum_{j=1}^{d}\left\|g_{j}\right\|^{2}} \leq \frac{\sqrt{d}}{2}
\end{aligned}
$$

as shown in the previous Example.

Taking $g_{0}=\frac{1}{\sqrt{2}} \Omega$ and $g_{k}=\frac{1}{\sqrt{2 d}} e_{j}$ for $j=1, \ldots, d$, we find that $\sum_{k=1}^{d}\left\|g_{k}\right\|^{2}=1$ while

$$
\left|\left\langle g_{0}, \sum_{j=1}^{d} B_{j} g_{j}\right\rangle\right|=\frac{1}{2 \sqrt{d}}\left|\left\langle\Omega, \sum_{j=1}^{d}\left\langle e_{j}, e_{j}\right\rangle \Omega\right\rangle=\right| \frac{\sqrt{d}}{2} .
$$

Example 10 For $d=1$ we find out that

$$
\frac{1}{2} w_{F}(T)=\frac{1}{2} w_{\mathrm{cl}}(T) \leq w_{B}(T) \leq w_{\mathrm{cl}}(T)=w_{F}(T)
$$

and $w_{B}(T)=\frac{1}{2} w_{F}(T)$ when $\mathcal{H}=\mathbb{C}, \quad T z:=a z, \quad a \in \mathbb{C}^{*}$. This shows that $w_{F}\left(T_{1}, \ldots, T_{d}\right) \neq w_{B}\left(T_{1}, \ldots, T_{d}\right)$ in general.

It was shown in $\left[9\right.$, Sect. 1] that $w_{F}(T)=w_{\mathrm{cl}}(T)$. Now, observe that

$$
\begin{aligned}
w_{B}(T) & =\sup \left\{\left|\left\langle g_{0}, T g_{1}\right\rangle\right|: g_{0}, g_{1} \in \mathcal{H},\left\|g_{0}\right\|^{2}+\left\|g_{1}\right\|^{2}=1\right\} \\
& \geq \sup \left\{\left|\left\langle g_{0}, T g_{0}\right\rangle\right|: g_{0} \in \mathcal{H},\left\|g_{0}\right\|^{2}=\frac{1}{2}\right\} \\
& =\sup \left\{\frac{1}{2}|\langle h, T h\rangle|: h:=\sqrt{2} g_{0} \in \mathcal{H},\|h\|^{2}=\frac{1}{2}\right\}=\frac{1}{2} w_{\mathrm{cl}}(T) .
\end{aligned}
$$

On the other hand, given $g_{0}, g_{1} \in \mathcal{H}$, with $\left\|g_{0}\right\|^{2}+\left\|g_{1}\right\|^{2}=1$, we can repeat the idea of Popescu, setting $f_{\theta}:=g_{0}+e^{\mathrm{i} \theta} g_{1}$ for $\theta \in[0,2 \pi]$. Then 


$$
\begin{aligned}
\int_{0}^{2 \pi}\left\|f_{\theta}\right\|^{2} d \theta & =\int_{0}^{2 \pi}\left\langle g_{0}+e^{\mathrm{i} \theta} g_{1}, g_{0}+e^{\mathrm{i} \theta} g_{1}\right\rangle d \theta \\
& =\left\|g_{0}\right\|^{2} \int_{0}^{2 \pi} d \theta+\left\langle g_{0}, g_{1}\right\rangle\left\|^{2} \int_{0}^{2 \pi} e^{-\mathrm{i} \theta} d \theta+\left\langle g_{1}, g_{0}\right\rangle\right\|^{2} \int_{0}^{2 \pi} e^{\mathrm{i} \theta} d \theta \\
& +\left\|g_{1}\right\|^{2} \int_{0}^{2 \pi} d \theta \\
& =2 \pi\left(\left\|g_{0}\right\|^{2}+\left\|g_{1}\right\|^{2}\right)=2 \pi
\end{aligned}
$$

and

$$
\begin{aligned}
\int_{0}^{2 \pi} & \left\langle f_{\theta}, T f_{\theta}\right\rangle e^{\mathrm{i} \theta} d \theta=\int_{0}^{2 \pi}\left\langle g_{0}+e^{\mathrm{i} \theta} g_{1}, T\left(g_{0}+e^{\mathrm{i} \theta} g_{1}\right)\right\rangle e^{\mathrm{i} \theta} d \theta \\
& =\int_{0}^{2 \pi}\left\langle g_{0}, T g_{0}\right\rangle e^{\mathrm{i} \theta} d \theta+\int_{0}^{2 \pi}\left\langle g_{0}, T g_{1}\right\rangle d \theta+\int_{0}^{2 \pi}\left\langle g_{1}, T g_{0}\right\rangle e^{2 \mathrm{i} \theta} d \theta+\int_{0}^{2 \pi}\left\langle g_{1}, T g_{1}\right\rangle e^{\mathrm{i} \theta} d \theta \\
& =2 \pi\left\langle g_{0}, T g_{1}\right\rangle .
\end{aligned}
$$

So, using the fact that $|\langle h, T h\rangle| \leq w_{c l}(T)\|h\|^{2}$, we get

$$
\left|\left\langle g_{0}, T g_{1}\right\rangle\right| \leq \frac{1}{2 \pi} \int_{0}^{2 \pi}\left|\left\langle f_{\theta}, T f_{\theta}\right\rangle e^{\mathrm{i} \theta}\right| d \theta \leq \frac{1}{2 \pi} w_{\mathrm{cl}}(T) \int_{0}^{2 \pi}\left\|f_{\theta}\right\|^{2} d \theta=w_{\mathrm{cl}}(T) .
$$

For the special choice $\mathcal{H}=\mathbb{C}$ and $T_{a} z:=a z$ for some fixed $a \in \mathbb{C}^{*}$, we get $w_{B}\left(T_{a}\right)=\frac{1}{2} w_{\mathrm{cl}}\left(T_{a}\right)$. Indeed,

$$
\begin{aligned}
w_{B}\left(T_{a}\right) & =\sup \left\{|\langle z, a w\rangle|:\|z\|^{2}+\|w\|^{2}=1\right\}=\left|\begin{array}{c}
z=e^{\mathrm{i} \phi} \sin t, w=e^{\mathrm{i} \psi} \cos t \\
\phi, \psi, t \in[0,2 \pi]
\end{array}\right| \\
& =\frac{|a|}{2} \sup \{|\sin (2 t)|, t \in[0,2 \pi]\}=\frac{|a|}{2}=\frac{w_{\mathrm{cl}}\left(T_{a}\right)}{2} .
\end{aligned}
$$

It is an open problem to check if the equality $w_{B}(T)=w_{F}(T)$ can hold.

We end this Section with an observation that the joint boolean spectral radius degenerates.

Proposition 11 The joint boolean spectral radius is always 0.

Proof Since the boolean creation operators satisfy $B_{j}^{*} B_{k}^{*}=0$ for any $j, k$, we have

$$
\begin{aligned}
r_{B}\left(T_{1}, \ldots, T_{d}\right) & =r\left(B_{1}^{*} \otimes T_{1}^{*}+\ldots B_{d}^{*} \otimes T_{d}^{*}\right)=\lim _{m \rightarrow \infty}\left\|\left(\sum_{j=1}^{d} B_{j}^{*} \otimes T_{j}^{*}\right)^{m}\right\|^{\frac{1}{m}} \\
& =\lim _{m \rightarrow \infty}\left\|\sum_{j_{1}, \ldots, j_{m}=1}^{d} B_{j_{1}}^{*} \ldots B_{j_{m}}^{*} \otimes T_{j_{1}}^{*} \ldots T_{j_{m}}^{*}\right\|^{\frac{1}{m}}=0 .
\end{aligned}
$$




\section{Joint monotone numerical and spectral radii}

We consider the model of the monotone independence on the discrete weakly monotone Fock space $\mathcal{F}_{W M}(H)$, defined in [13]. This space is built upon a $d$ dimensional Hilbert space $H$ with a given orthonormal basis $\left\{e_{j}: 1 \leq j \leq d\right\}$, as a closed subspace of the full Fock space $\mathcal{F}(H)$, spanned by the vacuum vector $\Omega:=e_{0}$ and the simple tensors $e_{j_{k}} \otimes \ldots \otimes e_{j_{1}}$, where the indices are in weakly monotonic order: $1 \leq j_{1} \leq \ldots \leq j_{k}$. By the standard convention we identify $e_{0} \otimes h=$ $h \otimes e_{0}=h$ for any $h \in \mathcal{F}_{W M}(H)$.

The creation operator $M_{j}^{*}$ by the vector $e_{j}$ is defined as follows:

$$
\begin{aligned}
& M_{j}^{*}\left(e_{j_{k}} \otimes \ldots \otimes e_{j_{1}}\right)=e_{j} \otimes e_{j_{k}} \otimes \ldots \otimes e_{j_{1}} \quad \text { if } \quad j \geq j_{k} \geq \ldots \geq j_{1} \geq 1, \\
& M_{j}^{*}\left(e_{j_{k}} \otimes \ldots \otimes e_{j_{1}}\right)=0 \quad \text { if } \quad j<j_{k} .
\end{aligned}
$$

The annihilation operator is defined as

$$
M_{j}\left(e_{j}\right)=\Omega, \quad M_{j}\left(e_{j_{k}} \otimes \ldots \otimes e_{j_{1}}\right)=\delta_{j j_{k}} e_{j_{k-1}} \otimes \ldots \otimes e_{j_{1}},
$$

and they are mutually adjoint: $\left(M_{k}\right)^{\star}=M_{k}^{*}$.

It is useful to introduce the following orthogonal projections. By definition $P_{0}$ is the orthogonal projection onto $e_{0}=\Omega$ and for $m \geq 1$ :

$$
\begin{aligned}
P_{m}\left(e_{j_{k}} \otimes \ldots \otimes e_{j_{1}}\right) & =e_{j_{k}} \otimes \ldots \otimes e_{j_{1}} \quad \text { if } \quad m=j_{k} \geq \ldots \geq j_{1} \geq 1 \\
P_{m}\left(e_{j_{k}} \otimes \ldots \otimes e_{j_{1}}\right) & =0 \quad \text { if } \quad m \neq j_{k} \quad \text { and } m \geq 1 \\
Q_{m} & :=P_{0}+P_{1}+\ldots+P_{m} .
\end{aligned}
$$

Then $Q_{m}$ is the orthogonal projection onto the span of $e_{0}=\Omega$ and vectors of the form $e_{j_{k}} \otimes \ldots \otimes e_{j_{1}}$ with $j_{k} \leq m$, and $P_{m}$ is the orthogonal projection onto the span of vectors of the form $e_{j_{k}} \otimes \ldots \otimes e_{j_{1}}$ with $j_{k}=m, k \geq 1$.

The weakly monotone creation and annihilation operators satisfy the following commutation relations

$$
\begin{gathered}
M_{j}^{*} M_{k}^{*}=M_{k} M_{j}=0 \quad \text { if } \quad j<k \\
M_{j} M_{k}^{*}=0 \quad \text { if } \quad j \neq k \\
M_{k} M_{k}^{*}=Q_{k} \quad \text { for } \quad 1 \leq k \leq d \\
M_{k}^{*} M_{k}=P_{k} \quad \text { for } \quad 1 \leq k \leq d .
\end{gathered}
$$

In particular, for $j<k$ we have

$$
M_{j} M_{k} M_{k}^{*} M_{j}^{*}=M_{j} Q_{k} M_{j}^{*}=Q_{j} .
$$

Remark 12 The monotone creation operators are bounded and generate *subalgebras which are monotone independent in the sense of Muraki [7, 8] (for the proof see [13] and [4]). 
In this setting the joint monotone numerical radius is defined as

$$
w_{M}\left(T_{1}, \ldots, T_{d}\right):=w_{\mathrm{cl}}\left(M_{1}^{*} \otimes T_{1}^{*}+\ldots M_{d}^{*} \otimes T_{d}^{*}\right)
$$

and the joint monotone spectral radius of $\left(T_{1}, \ldots, T_{d}\right)$ is defined as

$$
r_{M}\left(T_{1}, \ldots, T_{d}\right):=r_{\mathrm{cl}}\left(M_{1}^{*} \otimes T_{1}^{*}+\ldots M_{d}^{*} \otimes T_{d}^{*}\right) .
$$

To provide explicit formulas for the joint monotone numerical and spectral radii we introduce some operations on weakly monotone sequences. For each $k \in \mathbb{N}_{0}:=$ $\mathbb{N} \cup\{0\}$ we define

$$
\begin{aligned}
\mathcal{M}_{k} & :=\left\{\left(i_{k}, \ldots, i_{1}\right) \in \mathbb{N}^{k}: d \geq i_{k} \geq \ldots \geq i_{1} \geq 1\right\}, k \geq 1 \\
\mathcal{M}_{0} & :=\{0\} \\
\mathcal{M}_{-1} & :=\{\emptyset\}
\end{aligned}
$$

Note that each $\mathcal{M}_{k}$ with $k \geq 1$ is finite with cardinality $\left(\begin{array}{c}k+d-1 \\ k\end{array}\right)$. We also set $\mathcal{M}:=\bigcup_{k \geq 0} \mathcal{M}_{k}$ to be the union of all $\mathcal{M}_{k}$ for $k \geq 0$ and $\widetilde{\mathcal{M}}:=\mathcal{M} \cup \mathcal{M}_{-1}$.

There is a natural comparison relation $R$ of the sequences from $\mathcal{M}$, namely for $\alpha=\left(i_{k}, \ldots, i_{1}\right) \in \mathcal{M}_{k}$ and $\beta:=\left(j_{m}, \ldots, j_{1}\right) \in \mathcal{M}_{m}$ with $k, m \geq 1$ we put

$$
\begin{array}{lll}
(\alpha, \beta) \in R & \text { if } \quad i_{1} \geq j_{m} \\
(\alpha, \beta) \notin R & \text { if } \quad i_{1}<j_{m} \\
(\alpha, 0) \in R & \text { if } \quad \alpha \in \mathcal{M}_{k}, k \geq 1 .
\end{array}
$$

Observe that this relation is neither symmetric nor antisymmetric.

For convenience we shall write $\alpha \geq \beta$ iff $(\alpha, \beta) \in R$.

Definition 13 The weakly monotone concatenation of such sequences is defined on $\widetilde{\mathcal{M}}$ as follows: Let $\alpha=\left(i_{k}, \ldots, i_{1}\right) \in \mathcal{M}_{k}$ and $\beta:=\left(j_{m}, \ldots, j_{1}\right) \in \mathcal{M}_{m}$ with $k, m \geq 1$, then

$$
\begin{aligned}
\alpha \beta & :=\left(i_{k}, \ldots, i_{1}, j_{m}, \ldots, j_{1}\right) \quad \text { if } \quad \alpha \geq \beta \\
\alpha \beta & :=\emptyset \quad \text { if } \quad(\alpha, \beta) \notin R, \\
0 \alpha=\alpha 0 & :=\alpha \\
\emptyset \alpha=\alpha \emptyset & :=\emptyset .
\end{aligned}
$$

If $\alpha \in \mathcal{M}_{k}, \beta \in \mathcal{M}_{m}$ with $k, m \geq 0$ and $\alpha \geq \beta$, then $\alpha \beta \in \mathcal{M}_{k+m}$.

In particular, for $j \in \mathcal{M}_{1}$ and $\alpha=\left(i_{k}, \ldots, i_{1}\right) \in \mathcal{M}_{k}$ we shall have $j \alpha=$ $\left(j, i_{k}, \ldots, i_{1}\right)$ if $j \geq \alpha$. As one can see for this concatenation 0 plays the role of neutral element and the empty set $\emptyset$ behaves like 0 in multiplication of numbers.

Then, with the notation 


$$
e_{\alpha}=\left\{\begin{array}{l}
0 \text { if } \alpha=\emptyset, \\
\Omega \text { if } \alpha \in \mathcal{M}_{0}, \\
e_{i_{k}} \otimes \ldots \otimes e_{i_{1}} \text { if } \alpha=\left(i_{k}, \ldots, i_{1}\right) \in \mathcal{M}_{k}, k \geq 1,
\end{array}\right.
$$

the set $\left\{e_{\alpha}: \alpha \in \mathcal{M}\right\}$ is an orthonormal basis of $\mathcal{F}_{W M}(H)$. We shall extend this notation to the operators: if $T_{1}, \ldots, T_{d} \in B(\mathcal{H})$ are given and $\alpha \in \mathcal{M}$, then

$$
T_{\alpha}=\left\{\begin{array}{c}
I \text { if } \alpha \in \mathcal{M}_{0}, \\
T_{i_{k}} \otimes \ldots \otimes T_{i_{1}} \text { if } \alpha=\left(i_{k}, \ldots, i_{1}\right) \in \mathcal{M}_{k}, k \geq 1 .
\end{array}\right.
$$

Now we are ready to describe the explicit formula for the joint monotone numerical radius of $d$-tuple of operators.

Proposition 14 Let $\left(T_{1}, \ldots, T_{d}\right)$ be a d-tuple of bounded operators on a Hilbert space $\mathcal{H}$. Their joint monotone numerical radius can be computed as

$$
w_{M}\left(T_{1}, \ldots, T_{d}\right)=\sup \left\{\left|\sum_{j=1}^{d} \sum_{\alpha \in \mathcal{M}}\left\langle g_{\alpha} \mid T_{j} g_{j \alpha}\right\rangle\right|: g_{\alpha} \in \mathcal{H}, \sum_{\alpha \in \mathcal{M}}\left\|g_{\alpha}\right\|^{2}=1\right\} .
$$

Proof We express $h \in \mathcal{F}_{M}(H) \otimes \mathcal{H}$ as $h=\sum_{\alpha \in \mathcal{M}} e_{\alpha} \otimes g_{\alpha}$ with $g_{\alpha} \in \mathcal{H}$. Then

$$
\begin{aligned}
& w_{M}\left(T_{1}, \ldots, T_{d}\right)=\sup \left\{\left|\left\langle\sum_{j=1}^{d} M_{j}^{*} \otimes T_{j}^{*} h, h\right\rangle\right|: h \in \mathcal{F}_{M}(H) \otimes \mathcal{H},\|h\|=1\right\} \\
& \quad=\sup \left\{\left|\left\langle\sum_{j=1}^{d} M_{j}^{*} \otimes T_{j}^{*}\left(\sum_{\alpha \in \mathcal{M}} e_{\alpha} \otimes g_{\alpha}\right),\left(\sum_{\beta \in \mathcal{M}} e_{\beta} \otimes g_{\beta}\right)\right\rangle\right|: g_{\alpha} \in h,\left\|\sum_{\alpha \in \mathcal{M}} e_{\alpha} \otimes g_{\alpha}\right\|=1\right\} .
\end{aligned}
$$

Since

$$
\begin{aligned}
\left\|\sum_{\alpha \in \mathcal{M}} e_{\alpha} \otimes g_{\alpha}\right\|^{2} & =\left\langle\sum_{\alpha \in \mathcal{M}} e_{\alpha} \otimes g_{\alpha}, \sum_{\beta \in \mathcal{M}} e_{\beta} \otimes g_{\beta}\right\rangle \\
& =\sum_{\alpha, \beta \in \mathcal{M}}\left\langle e_{\alpha}, e_{\beta}\right\rangle\left\langle g_{\alpha}, g_{\beta}\right\rangle=\sum_{\alpha \in \mathcal{M}}\left\|g_{\alpha}\right\|^{2},
\end{aligned}
$$

and

$$
\begin{aligned}
& \left\langle\sum_{j=1}^{d} M_{j}^{*} \otimes T_{j}^{*}\left(\sum_{\alpha \in \mathcal{M}} e_{\alpha} \otimes g_{\alpha}\right),\left(\sum_{\beta \in \mathcal{M}} e_{\beta} \otimes g_{\beta}\right)\right\rangle= \\
& =\sum_{j=1}^{d} \sum_{\alpha, \beta \in \mathcal{M}}\left\langle M_{j}^{*} e_{\alpha}, e_{\beta}\right\rangle\left\langle T_{j}^{*} g_{\alpha}, g_{\beta}\right\rangle \\
& =\sum_{j=1}^{d} \sum_{\alpha, \beta \in \mathcal{M}}\left\langle e_{j \alpha}, e_{\beta}\right\rangle\left\langle T_{j}^{*} g_{\alpha}, g_{\beta}\right\rangle=\sum_{j=1}^{d} \sum_{\alpha \in \mathcal{M}}\left\langle g_{\alpha}, T_{j} g_{j \alpha}\right\rangle,
\end{aligned}
$$

the formula (11) follows. 
Note that in the summation over $\alpha \in \mathcal{M}$ the nonzero terms might be only for those $\alpha$ for which $j \alpha \neq \emptyset$, i.e. when $j \geq i_{k}$ if $\alpha=\left(i_{k}, \ldots, i_{1}\right)$. For example, if $j=1$ then it must be $\alpha=0$ or $\alpha=(1, \ldots, 1)$, so in this case the summation over $\alpha \in \mathcal{M}$ reduces to the summation over $k=0,1, \ldots$, the length of the sequence of 1 's.

To study the properties of the joint monotone numerical radius we need the following lemma.

Lemma 1 For any $T_{1}, \ldots, T_{d} \in B(\mathcal{H})$ we have

$$
\left\|\sum_{j=1}^{d} M_{j}^{*} \otimes T_{j}^{*}\right\|=\max _{1 \leq m \leq d}\left\|\sum_{j=m}^{d} T_{j} T_{j}^{*}\right\|^{\frac{1}{2}} .
$$

Proof Using (7) and (8) and the definition of $Q_{j}$, we observe that

$$
\begin{aligned}
\left\|\sum_{j=1}^{d} M_{j}^{*} \otimes T_{j}^{*}\right\| & =\left\|\left(\sum_{j=1}^{d} M_{j} \otimes T_{j}\right)\left(\sum_{k=1}^{d} M_{k}^{*} \otimes T_{k}^{*}\right)\right\|^{\frac{1}{2}}=\|\sum_{j, k=1}^{d} \underbrace{M_{j} M_{k}^{*}}_{=\delta_{j k} Q_{j}} \otimes T_{j} T_{k}^{*}\|^{\frac{1}{2}} \\
& =\left\|\sum_{j=1}^{d} Q_{j} \otimes T_{j} T_{j}^{*}\right\|^{\frac{1}{2}}=\left\|\sum_{j=1}^{d}\left(\sum_{m=0}^{j} P_{m}\right) \otimes T_{j} T_{j}^{*}\right\|^{\frac{1}{2}} \\
& =\left\|P_{0} \otimes \sum_{j=1}^{d} T_{j} T_{j}^{*}+\sum_{m=1}^{d} P_{m} \otimes \sum_{j=m}^{d} T_{j} T_{j}^{*}\right\|^{\frac{1}{2}}
\end{aligned}
$$

Now, we use the mutual orthogonality of the orthogonal projections $P_{0}, P_{1}, \ldots, P_{d}$ to get

$$
\left\|\sum_{j=1}^{d} M_{j}^{*} \otimes T_{j}^{*}\right\|=\max _{1 \leq m \leq d}\left\|\sum_{j=m}^{d} T_{j} T_{j}^{*}\right\|^{\frac{1}{2}}
$$

In analogy to Proposition 6 we have the following properties of the joint monotone numerical radius.

Proposition 15 We have

$(i)_{M} \quad w_{M}\left(T_{1}, \ldots, T_{d}\right)=0$ if and only if $T_{1}=\ldots=T_{d}=0$,

$(\text { ii })_{M} \quad \frac{1}{2} \max _{1 \leq m \leq d}\left\|\sum_{j=m}^{d} T_{j} T_{j}^{*}\right\|^{\frac{1}{2}} \leq w_{M}\left(T_{1}, \ldots, T_{d}\right) \leq \max _{1 \leq m \leq d}\left\|\sum_{j=m}^{d} T_{j} T_{j}^{*}\right\|^{\frac{1}{2}}$,

$(\text { iii })_{M} \quad w_{M}\left(X^{*} T_{1} X, \ldots, X^{*} T_{d} X\right) \leq\|X\|^{2} w_{M}\left(T_{1}, \ldots, T_{d}\right)$ for any bounded operator $X: \mathcal{H} \rightarrow \mathcal{K}$. 
Proof $\operatorname{Ad}(i)_{M}$. As in the proof of Proposition $6, w_{M}\left(T_{1}, \ldots, T_{d}\right)=0$ implies $\sum_{j=1}^{d} M_{j}^{*} \otimes T_{j}^{*}=0$. Hence for any $h=\sum_{k=0}^{d} e_{k} \otimes g_{k} \in \mathcal{F}_{B}(H) \otimes \mathcal{H}$, one gets

$$
0=\sum_{j=1}^{d} M_{j}^{*} \otimes T_{j}^{*} h=\sum_{j=1}^{d} \sum_{k=0}^{d} M_{j}^{*} e_{k} \otimes T_{j}^{*} g_{k}=\sum_{k=0}^{d} \sum_{j>k} e_{j} \otimes e_{k} \otimes T_{j}^{*} g_{0} .
$$

Hence $T_{j}=0$ for any $j=1, \ldots, d$.

Ad $(i i)_{M}$. Due to $w_{c l}(T) \leq\|T\|$ and Lemma 1 we have

$$
w_{M}\left(T_{1}, \ldots, T_{d}\right)=w_{\mathrm{cl}}\left(\sum_{j=1}^{d} M_{j}^{*} \otimes T_{j}^{*}\right) \leq\left\|\sum_{j=1}^{d} M_{j}^{*} \otimes T_{j}^{*}\right\|=\max _{1 \leq m \leq d}\left\|\sum_{j=m}^{d} T_{j} T_{j}^{*}\right\|^{\frac{1}{2}} .
$$

The other part follows from $\frac{1}{2}\|T\| \leq w_{c l}(T)$.

Ad $(\text { iii })_{M}$. Let $X: \mathcal{H} \rightarrow \mathcal{K}$ be a bounded operator and let $g_{0}, \ldots, g_{d} \in \mathcal{H}$ satisfy $\sum_{k=0}^{d}\left\|g_{k}\right\|^{2}=1$. Define $C:=\sqrt{\sum_{k=0}^{d}\left\|X g_{k}\right\|^{2}}$ and $h_{k}:=\frac{1}{C} X g_{k}, k=0,1, \ldots, d$. Then $\sum_{k=0}^{d}\left\|h_{k}\right\|^{2}=1$ and $C \leq\|X\|$. Consequently,

$$
\begin{aligned}
w_{M}\left(X^{*} T_{1} X, \ldots, X^{*} T_{d} X\right) & =\sup \left\{\left|\left\langle X g_{0}, \sum_{j=1}^{d} T_{j} X g_{j}\right\rangle\right|: \sum_{j=0}^{d}\left\|g_{j}\right\|^{2}=1\right\} \\
& \leq \sup \left\{\left|\left\langle C h_{0}, \sum_{j=1}^{d} T_{j} C h_{j}\right\rangle\right|: \sum_{j=0}^{d}\left\|h_{j}\right\|^{2}=1\right\} \\
& =C^{2} w_{M}\left(T_{1}, \ldots, T_{d}\right) \leq\|X\|^{2} w_{M}\left(T_{1}, \ldots, T_{d}\right) .
\end{aligned}
$$

Remark 16 The properties $(i)_{M},(i i)_{M}$ and $(i)_{M}$ show that the joint monotone numerical radius is a norm on $B(\mathcal{H})^{d}$, which, by $(i i)_{M}$, is actually equivalent to the maximum, over $1 \leq m \leq d$, of the operator norms of the operator row matrices $\left[T_{m}, \ldots, T_{d}\right]$.

Example 17 For $d=1$ and $T \in B(\mathcal{H})$ we get $w_{M}(T)=w_{F}(T)=w_{\mathrm{cl}}(T)$.

This follows immediately from the fact that for $d=1$ the set $\mathcal{M}_{k}$ consists of the unique term $(1, \ldots, 1)$ ( $k$-times). Hence,

$$
w_{M}(T)=\sup \left\{\left|\sum_{k=0}^{\infty}\left\langle g_{k}, T g_{k+1}\right\rangle\right|: \sum_{k=0}^{\infty}\left\|g_{k}\right\|^{2}=1\right\},
$$

which is exactly the same as the joint free numerical radius for a single operator, and which in turn was shown to be equal to the classical numerical radius (see [9, Sect. 1]).

The joint monotone spectral radius can be computed similarly to the (free) joint spectral radius of Popescu. 
Proposition 18 The joint monotone spectral radius satisfies

$$
r_{M}\left(T_{1}, \ldots, T_{d}\right)=\lim _{k \rightarrow \infty} \max _{1 \leq m \leq d}\left\|\sum_{j=m}^{d} \sum_{\alpha \in \mathcal{M}_{k}^{j}} T_{\alpha} T_{\alpha}^{*}\right\|^{1 / 2 k},
$$

where

$$
\mathcal{M}_{k}^{j}:=\left\{\alpha=\left(i_{k}, \ldots, i_{1}\right) \in \mathcal{M}_{k}: i_{k} \geq \ldots \geq i_{1}=j\right\}
$$

is a set of monotone sequences of length $k$, starting from $j$.

Proof First observe that

$$
\begin{aligned}
\left(\sum_{j=1}^{d} M_{j}^{*} \otimes T_{j}^{*}\right)^{k} & =\sum_{j_{1}, \ldots, j_{k}=1}^{d} M_{j_{k}}^{*} \ldots M_{j_{1}}^{*} \otimes T_{j_{k}}^{*} \ldots T_{j_{1}}^{*} \\
& =\sum_{j_{k} \geq \ldots \geq j_{1} \geq 1} M_{j_{k}}^{*} \ldots M_{j_{1}}^{*} \otimes T_{j_{k}}^{*} \ldots T_{j_{1}}^{*}=\sum_{\alpha \in \mathcal{M}_{k}} M_{\alpha}^{*} \otimes T_{\alpha}^{*},
\end{aligned}
$$

due to (6). This also implies

$$
\begin{aligned}
& \left(\sum_{m=1}^{d} M_{m} \otimes T_{m}\right)^{k}\left(\sum_{j=1}^{d} M_{j}^{*} \otimes T_{j}^{*}\right)^{k} \\
& =\sum_{m_{1}, \ldots, m_{k}=1}^{d} \sum_{j_{1}, \ldots, j_{k}=1}^{d} M_{m_{1}} \ldots M_{m_{k}} M_{j_{k}}^{*} \ldots M_{j_{1}}^{*} \otimes T_{m_{1}} \ldots T_{m_{k}} T_{j_{k}}^{*} \ldots T_{j_{1}}^{*} \\
& =\sum_{1 \leq m_{1} \leq \ldots \leq m_{k} j_{k} \geq \ldots \geq j_{1} \geq 1} \sum_{m_{1}} \ldots M_{m_{k}} M_{j_{k}}^{*} \ldots M_{j_{1}}^{*} \otimes T_{m_{1}} \ldots T_{m_{k}} T_{j_{k}}^{*} \ldots T_{j_{1}}^{*} \\
& =\sum_{j_{k} \geq \ldots \geq j_{1} \geq 1} M_{j_{1}} \ldots M_{j_{k}} M_{j_{k}}^{*} \ldots M_{j_{1}}^{*} \otimes T_{j_{1}} \ldots T_{j_{k}} T_{j_{k}}^{*} \ldots T_{j_{1}}^{*} \\
& =\sum_{j_{k} \geq \ldots \geq j_{1} \geq 1} Q_{j_{1}} \otimes T_{j_{1}} \ldots T_{j_{k}} T_{j_{k}}^{*} \ldots T_{j_{1}}^{*} \\
& =\sum_{m=1}^{d} Q_{m} \otimes T_{m}\left(\sum_{m \leq j_{2} \leq \ldots \leq j_{k} \leq d} T_{j_{2}} \ldots T_{j_{k}} T_{j_{k}}^{*} \ldots T_{j_{2}}^{*}\right) T_{m}^{*} \\
& =\sum_{m=1}^{d}\left(Q_{1}+P_{2}+\ldots+P_{m}\right) \otimes T_{m}\left(\sum_{m \leq j_{2} \leq \ldots \leq j_{k} \leq d} T_{j_{2}} \ldots T_{j_{k}} T_{j_{k}}^{*} \ldots T_{j_{2}}^{*}\right) T_{m}^{*} \\
& =Q_{1} \otimes T_{1}\left(\sum_{1 \leq j_{2} \leq \ldots \leq j_{k} \leq d} T_{j_{2}} \ldots T_{j_{k}} T_{j_{k}}^{*} \ldots T_{j_{2}}^{*}\right) T_{1}^{*}+ \\
& +\sum_{m=2}^{d} P_{m} \otimes \sum_{i=m}^{d} T_{i}\left(\sum_{i \leq j_{2} \leq \ldots \leq j_{k} \leq d} T_{j_{2}} \ldots T_{j_{k}} T_{j_{k}}^{*} \ldots T_{j_{2}}^{*}\right) T_{i}^{*}
\end{aligned}
$$


Since the projections $Q_{1}, P_{2}, \ldots, P_{d}$ are mutually orthogonal, we obtain

$$
\begin{aligned}
& \left\|\left(\sum_{m=1}^{d} M_{m} \otimes T_{m}\right)^{k}\left(\sum_{j=1}^{d} M_{j}^{*} \otimes T_{j}^{*}\right)^{k}\right\| \\
& \quad=\max _{1 \leq m \leq d}\left\|\sum_{j_{1}=m}^{d} \sum_{j_{1} \leq j_{2} \leq \ldots \leq j_{k} \leq d} T_{j_{1}} T_{j_{2}} \ldots T_{j_{k}} T_{j_{k}}^{*} \ldots T_{j_{2}}^{*} T_{j_{1}}^{*}\right\| .
\end{aligned}
$$

Therefore we can write

$$
\begin{aligned}
& r_{M}\left(T_{1}, \ldots, T_{d}\right)=r_{c l}\left(\sum_{j=1}^{d} M_{j}^{*} \otimes T_{j}^{*}\right)=\lim _{k \rightarrow \infty}\left\|\left(\sum_{j=1}^{d} M_{j}^{*} \otimes T_{j}^{*}\right)^{k}\right\|^{\frac{1}{k}} \\
& =\lim _{k \rightarrow \infty}\left\|\left(\sum_{m=1}^{d} M_{m} \otimes T_{m}\right)^{k}\left(\sum_{j=1}^{d} M_{j}^{*} \otimes T_{j}^{*}\right)^{k}\right\|^{1 / 2 k} \\
& =\lim _{k \rightarrow \infty} \max _{1 \leq m \leq d}\left\|\sum_{j_{1}=m}^{d} \sum_{j_{1} \leq j_{2} \leq \ldots \leq j_{k} \leq d} T_{j_{1}} T_{j_{2}} \ldots T_{j_{k}} T_{j_{k}}^{*} \ldots T_{j_{2}}^{*} T_{j_{1}}^{*}\right\|^{1 / 2 k} \\
& \quad=\lim _{k \rightarrow \infty} \max _{1 \leq m \leq d}\left\|\sum_{j=m}^{d} \sum_{\alpha \in \mathcal{M}_{k}^{j}} T_{\alpha} T_{\alpha}^{*}\right\|^{1 / 2 k} .
\end{aligned}
$$

\subsection{Example: joint monotone numerical radius for weakly monotone annihilation operators}

We shall compute the joint monotone numerical radius for $d$-tuple of weakly monotone annihilators $M_{1}, \ldots, M_{d}$. An upper bound for $w_{M}\left(M_{1}, \ldots, M_{d}\right)$ is easily obtained.

Proposition 19 For any $d \geq 2$ we have

$$
w_{M}\left(M_{1}, \ldots, M_{d}\right) \leq d
$$

Proof Using the fact that the norm of any creation operator $M_{j}$ is 1 and the CauchySchwarz inequality, we have the following

$$
\begin{aligned}
\left|\sum_{j=1}^{d} \sum_{\alpha \in \mathcal{M}}\left\langle g_{\alpha} \mid M_{j} g_{j \alpha}\right\rangle\right| & \leq \sum_{j=1}^{d} \sum_{\alpha \in \mathcal{M}}\left|\left\langle M_{j}^{*} g_{\alpha} \mid g_{j \alpha}\right\rangle\right| \leq \sum_{j=1}^{d} \sum_{\alpha \in \mathcal{M}}\left\|g_{\alpha}\right\|\left\|g_{j \alpha}\right\| \\
& \leq \sum_{j=1}^{d}\left(\sum_{\alpha \in \mathcal{M}}\left\|g_{\alpha}\right\|^{2}\right)^{\frac{1}{2}}\left(\sum_{\alpha \in \mathcal{M}}\left\|g_{j \alpha}\right\|^{2}\right)^{\frac{1}{2}} \leq \sum_{j=1}^{d} 1=d
\end{aligned}
$$


for any family $\left(g_{\alpha}\right)_{\alpha \in \mathcal{M}}$ satisfying $\sum_{\alpha \in \mathcal{M}}\left\|g_{\alpha}\right\|^{2}=1$.

This is a rough estimate and the following calculations show that perhaps the optimal one would be related to $\sqrt{d}$. To support this claim, we provide lower estimates of that sort.

It will be instructive to treat first the case $d=2$.

Proposition 20 For two weakly monotone annihilation operators $T_{1}=M_{1}$ and $T_{2}=M_{2}$, we have $w_{M}\left(M_{1}, M_{2}\right) \geq \frac{4}{3 \sqrt{3}} \sqrt{2} \approx 0,77 \cdot \sqrt{2}$.

Proof For the proof we shall analyze in details the expression

$$
\sum_{j=1}^{2} \sum_{\alpha}\left\langle g_{\alpha} \mid M_{j} g_{j \alpha}\right\rangle \text {. }
$$

For this purpose we shall use the notation $\alpha=[k, m]$ if $\alpha=(2, \ldots, 2,1, \ldots 1)$ is a sequence of $m$ times the digit 2 followed by $k$ times the digit 1 . Then we have explicit formulas for the composition $j \alpha$ with $j=1,2$, namely $2[k, m]=[k, m+1]$ if $k, m \geq 0,1[k, 0]=[k+1,0]$ if $k \geq 1$ and $1[k, m]=0$ if $m \geq 1$.

We shall also denote the orthonormal basis $e_{\alpha}=e_{m, k}$ if $\alpha=[k, m]$; in particular $\left\langle e_{s, r} \mid e_{u, t}\right\rangle=1$ if and only if $s=u, r=t$. Each vector $g_{[k, m]}$ can be written in the orthonormal basis as $g_{[k, m]}=\sum_{r, s \geq 0} g_{[k, m]}^{r, s} e_{s, r}$ and then we get

$$
\sum_{\alpha}\left\|g_{\alpha}\right\|^{2}=\sum_{k, m, r, s \geq 0}\left|g_{[k, m]}^{r, s}\right|^{2}
$$

and

$$
\begin{aligned}
\sum_{j=1}^{2} \sum_{\alpha}\left\langle g_{\alpha} \mid M_{j} g_{j \alpha}\right\rangle & =\sum_{j=1}^{2} \sum_{k, m \geq 0} \sum_{r, s \geq 0} \sum_{t, u \geq 0} g_{[k, m]}^{r, s} g_{j[k, m]}^{t, u}\left\langle e_{s, r} \mid M_{j} e_{u, t}\right\rangle \\
& =\sum_{j=1}^{2} \sum_{k, m \geq 0} \sum_{r, s \geq 0} \sum_{t, u \geq 0} g_{[k, m]}^{r, s} g_{j[k, m]}^{t, u}\left\langle M_{j}^{*} e_{s, r} \mid e_{u, t}\right\rangle .
\end{aligned}
$$

We split this summation into two parts.

1. For $j=1$ we have necessarily $m=0$ and $\left\langle M_{1}^{*} e_{s, r} \mid e_{u, t}\right\rangle=\left\langle e_{0, r+1} \mid e_{u, t}\right\rangle$ if $s=0$ and $\left\langle M_{j}^{*} e_{s, r} \mid e_{u, t}\right\rangle=0$ if $s \geq 1$. Hence for $j=1$ we get only the following nonzero term

$$
\sum_{k \geq 0} \sum_{r \geq 0} \sum_{t, u \geq 0} g_{[k, 0]}^{r, s} g_{[k+1,0]}^{t, u}\left\langle e_{0, r+1} \mid e_{u, t}\right\rangle=\sum_{k, r \geq 0} g_{[k, 0]}^{r, 0} g_{[k+1,0]}^{r+1,0} .
$$

2. For $j=2$ and $m \geq 0$ in a similar manner we get

$$
\left\langle M_{2}^{*} e_{s, r} \mid e_{u, t}\right\rangle=\left\langle e_{s+1, r} \mid e_{u, t}\right\rangle=1 \text { if and only if } u=s+1, t=r .
$$


Hence the nonzero term in (15) is

$$
\sum_{k \geq 0} \sum_{m \geq 1} \sum_{r \geq 0} \sum_{t, u \geq 0} g_{[k, m]}^{r, s} g_{[k, m+1]}^{t, u}\left\langle e_{s+1, r} \mid e_{u, t}\right\rangle=\sum_{k \geq 0} \sum_{m \geq 1} \sum_{r, s \geq 0} g_{[k, m]}^{r, s} g_{[k, m+1]}^{r, s+1} .
$$

So we get

$$
\begin{aligned}
\sum_{j=1}^{2} \sum_{\alpha}\left\langle g_{\alpha} \mid M_{j} g_{j \alpha}\right\rangle & =\sum_{j=1}^{2} \sum_{k, m \geq 0} \sum_{r, s \geq 0} \sum_{t, u \geq 0} g_{[k, m]}^{r, s} g_{j[k, m])}^{t, u}\left\langle M_{j}^{*} e_{s, r} \mid e_{u, t}\right\rangle \\
& =\sum_{k, r \geq 0} g_{[k, 0]}^{r, 0} g_{[k+1,0]}^{r+1,0}+\sum_{k, m \geq 0} \sum_{r, s \geq 0} g_{[k, m]}^{r, s} g_{[k, m+1]}^{r, s+1} .
\end{aligned}
$$

Now we consider special vectors, defined for a constant $x \in(0,1)$ and $k, m, r, s \geq 0$ :

$$
g_{[k, m]}^{r s}=\left\{\begin{array}{cc}
x^{m+k}, & \text { if } r=k, s=m \\
0 & \text { otherwise }
\end{array}\right.
$$

We have that in this case the quantity in (14) equals

$$
\sum_{\alpha \in \mathcal{M}}\left\|g_{\alpha}\right\|^{2}=\sum_{k, m, r, s=0}^{\infty}\left|g_{[k, m]}^{r s}\right|^{2}=\sum_{k, m=0}^{\infty} x^{2 k} x^{2 m}=\frac{1}{\left(1-x^{2}\right)^{2}} .
$$

On the other hand, we have

$$
\begin{aligned}
\sum_{j=1}^{2} & \sum_{\alpha}\left\langle g_{\alpha} \mid M_{j} g_{j \alpha}\right\rangle \\
& =\sum_{k \geq 0} x^{2 k+1}+\sum_{k \geq 0} \sum_{m \geq 0} x^{m+k} x^{m+k+1} \\
& =\frac{x}{1-x^{2}}+\frac{x}{\left(1-x^{2}\right)^{2}}=\frac{2 x-x^{3}}{\left(1-x^{2}\right)^{2}} .
\end{aligned}
$$

Hence maximizing the quotient of (18) by (17) we get the function $f(x):=2 x-x^{3}$, which in $(0,1)$ has the local maximum $\frac{4}{3} \sqrt{\frac{2}{3}}$ at $x=\sqrt{\frac{2}{3}}$.

To sum up, we have

$$
w_{M}\left(M_{1}, M_{2}\right) \geq \sup _{x \in(0,1)} f(x)=\frac{4}{3} \sqrt{\frac{2}{3}} .
$$

We are now ready to treat the general case.

Proposition 21 For $d \geq 3$ and the monotone annihilators $T_{j}=M_{j}, j=1, \ldots, d$, we have 


$$
w_{M}\left(M_{1}, \ldots, M_{j}\right) \geq \frac{5}{9} \sqrt{d}
$$

Proof We generalize the idea from the proof of the case $d=2$. For a fixed $d \geq 3$, any $\alpha \in \mathcal{M}$ is of the form

$$
\alpha=(\underbrace{d, \ldots, d}_{k_{d} \text {-times }}, \underbrace{d-1, \ldots, d-1}_{k_{d-1} \text {-times }}, \ldots, \underbrace{2, \ldots, 2}_{k_{2} \text {-times }}, \underbrace{1, \ldots, 1}_{k_{1}-\text { times }}),
$$

where $k_{d}, \ldots, k_{1} \geq 0$. In the sequel, such an element will be simply denoted by $\alpha=\left[k_{d}, \ldots, k_{1}\right]$. For arbitrary (but fixed) $x \in(0,1)$ we shall consider the vectors

$$
g_{\alpha}=g_{\left[k_{d}, \ldots, k_{1}\right]}:=x^{k_{d}+\ldots+k_{1}} e_{\alpha},
$$

for which we have

$$
\sum_{\alpha \in \mathcal{M}}\left\|g_{\alpha}\right\|^{2}=\sum_{k_{d}, \ldots, k_{1} \geq 0} x^{2\left(k_{d}+\ldots+k_{1}\right)}=\frac{1}{\left(1-x^{2}\right)^{d}} .
$$

Now, we would like to compute

$$
\sum_{j=1}^{d} \sum_{\alpha \in \mathcal{M}}\left\langle g_{\alpha} \mid M_{j} g_{j \alpha}\right\rangle
$$

For this purpose observe that, for a fixed $1 \leq j \leq d$, if $\alpha=\left[k_{d}, \ldots, k_{1}\right]$, then $j \alpha \neq \emptyset$ if and only if there are no indices bigger than $j$ in $\alpha$, that is if $k_{j+1}=0, \ldots, k_{d}=0$. In such case $j \alpha=\left[0, \ldots, 0, k_{j}+1, k_{j-1}, \ldots, k_{1}\right]$ and

$$
\begin{aligned}
\left\langle g_{\alpha} \mid M_{j} g_{j \alpha}\right\rangle & =\left\langle g_{\left[0, \ldots, 0, k_{j}, \ldots, k_{1}\right]} \mid M_{j} g_{\left[0, \ldots, 0, k_{j}+1, \ldots, k_{1}\right]}\right\rangle \\
& =\left\langle x^{k_{j}+\ldots+k_{1}} e_{\left[0, \ldots, 0, k_{j}, \ldots, k_{1}\right]} \mid x^{k_{j}+\ldots+k_{1}+1} M_{j} e_{\left[0, \ldots, 0, k_{j}+1, \ldots, k_{1}\right]}\right\rangle \\
& =x^{2\left(k_{j}+\ldots+k_{1}\right)+1}\left\langle e_{\left.0, \ldots, 0, k_{j}, \ldots, k_{1}\right]} \mid e_{\left[0, \ldots, 0, k_{j}, \ldots, k_{1}\right]}\right\rangle=x^{2\left(k_{j}+\ldots+k_{1}\right)+1} .
\end{aligned}
$$

Otherwise $j \alpha=\emptyset$ and $\left\langle g_{\alpha} \mid M_{j} g_{j \alpha}\right\rangle=0$.

This simplifies the expression (20) considerably:

$$
\sum_{j=1}^{d} \sum_{\alpha \in \mathcal{M}}\left\langle g_{\alpha} \mid M_{j} g_{j \alpha}\right\rangle=\sum_{j=1}^{d} \sum_{k_{j}, \ldots k_{1} \geq 0} x^{2\left(k_{j}+\ldots+k_{1}\right)+1}=\sum_{j=1}^{d} \frac{x}{\left(1-x^{2}\right)^{j}} .
$$

Taking into account the normalization (19) of the family $\left(g_{\alpha}\right)_{\alpha}$, we arrive to the problem of maximizing the function:

$$
f_{d}(x):=\frac{\sum_{j=1}^{d} \frac{x}{\left(1-x^{2}\right)^{j}}}{\frac{1}{\left(1-x^{2}\right)^{d}}}=\sum_{j=1}^{d} x\left(1-x^{2}\right)^{d-j}=x \sum_{k=0}^{d-1}\left(1-x^{2}\right)^{k}
$$

over $(0,1)$. Setting $y=1-x^{2}$ this reduces to the maximization of the function 


$$
g_{d}(y):=\sqrt{1-y} \sum_{k=0}^{d-1} y^{k}=\frac{1-y^{d}}{\sqrt{1-y}}
$$

over $(0,1)$.

Trying to resolve the optimization problem explicitly, one arrives to the equation

$$
(2 d-1) y^{d}-2 d y^{d-1}+1=0,
$$

which describes the critical points of $g_{d}$. We shall not go this way. We just observe that the value of $g_{d}$ at the points $y_{d}=1-\frac{1}{d}$ provides the required estimates. Namely,

$$
\sup _{y \in(0,1)} g(y) \geq g\left(1-\frac{1}{d}\right)=\sqrt{d}\left(1-\left(1-\frac{1}{d}\right)^{d}\right) \geq \frac{5}{9} \sqrt{d} \quad \text { for } \quad d \geq 3 .
$$

This shows that the numerical radius satisfies

$$
w_{M}\left(M_{1}, \ldots, M_{d}\right) \geq \sup _{x \in(0,1)} f(x)=\sup _{y \in(0,1)} g(y) \geq \frac{5}{9} \sqrt{d} .
$$

Let us remark that numerical calculations reveal that the point $y_{d}=1-\frac{1}{d}$ seems to be a good approximation of $y_{d, \max }$, at which the maximum of $g_{d}$ is achieved. Moreover, for $3 \leq d \leq 100$, the relative error appearing when $g_{d}\left(y_{d, \max }\right)$ is replaced by $g_{d}\left(y_{d}\right)$ is smaller than $2 \%$.

All these calculations and observations have led us to the formulation of the following statement.

Conjecture 1 There exists an absolute constant $c>0$ such that the joint monotone numerical radius of the d-tuple of weakly monotone annihilators equals

$$
w_{M}\left(M_{1}, \ldots, M_{d}\right)=c \sqrt{d} .
$$

\subsection{Example: joint monotone spectral radius for weakly monotone creation operators}

Using Proposition 18 we compute the joint monotone spectral radius of $d$ weakly monotone annihilation operators $M_{1}, \ldots, M_{d}$. In this case we have

$$
r_{M}\left(M_{1}, \ldots, M_{d}\right)=\lim _{k \rightarrow \infty} \max _{1 \leq m \leq d}\left\|\sum_{j=m}^{d} \sum_{\alpha \in \mathcal{M}_{k}^{j}} M_{\alpha} M_{\alpha}^{*}\right\|^{1 / 2 k}=1 .
$$

Indeed, let us denote by $c_{k}^{j}$ the cardinality of the set $\mathcal{M}_{k}^{j}$, see (13). Then $c_{j k}=\left|\mathcal{M}_{k}^{j}\right|=\left(\begin{array}{c}k+d-j \\ k-1\end{array}\right)$. Since for $\alpha \in \mathcal{M}_{k}^{j}$, we have $M_{\alpha} M_{\alpha}^{*}=Q_{j}$, the righthand side above can be written as 


$$
\begin{aligned}
& \lim _{k \rightarrow \infty} \max _{1 \leq m \leq d}\left\|\sum_{j=m}^{d} c_{k}^{j} \cdot Q_{j}\right\|^{1 / 2 k}=\lim _{k \rightarrow \infty} \max _{1 \leq m \leq d}\left\|\sum_{j=m}^{d} c_{k}^{j} \cdot\left(P_{0}+\sum_{i=1}^{j} P_{i}\right)\right\|^{1 / 2 k} \\
& =\lim _{k \rightarrow \infty} \max _{1 \leq m \leq d}\left\{\left\|Q_{m} \sum_{j=0}^{d-m} c_{k}^{m+j}\right\|^{1 / 2 k},\left\|\sum_{s=1}^{d-m} P_{m+s} \sum_{j=s}^{d-m} c_{k}^{m+j}\right\|^{1 / 2 k}\right\} \\
& =\lim _{k \rightarrow \infty} \max _{1 \leq m \leq d}\left\{\left\|Q_{m} \sum_{j=0}^{d-m} c_{k}^{m+j}\right\|^{1 / 2 k}, \max _{1 \leq s \leq d-m}\left\|P_{m+s} \sum_{j=s}^{d-m} c_{k}^{m+j}\right\|^{1 / 2 k}\right\} \\
& =\lim _{k \rightarrow \infty} \max _{1 \leq m \leq d}\left\{\left(\sum_{j=0}^{d-m} c_{k}^{m+j}\right)^{1 / 2 k}, \max _{1 \leq s \leq d-m}\left(\sum_{j=s}^{d-m} c_{k}^{m+j}\right)^{1 / 2 k}\right\}
\end{aligned}
$$

We see that the maximum is achieved for $m=1$ and the last expression equals

$$
\begin{aligned}
& \lim _{k \rightarrow \infty} \max _{1 \leq m \leq d} \max _{0 \leq s \leq d-m}\left\{\left(\sum_{j=s}^{d-m} c_{k}^{m+j}\right)^{1 / 2 k}\right\}=\lim _{k \rightarrow \infty}\left(\sum_{j=1}^{d} c_{k}^{j}\right)^{1 / 2 k} \\
&=\lim _{k \rightarrow \infty}\left(\sum_{j=1}^{d}\left(\begin{array}{c}
k+d-j \\
k-1
\end{array}\right)\right)^{1 / 2 k}=\lim _{k \rightarrow \infty}\left(\begin{array}{c}
k+d \\
k-1
\end{array}\right)^{1 / 2 k}=1 .
\end{aligned}
$$

\section{Concluding remarks and open problems}

This paper is a beginning of the studies of joint numerical and spectral radii related to creation operators independent in noncommutative sense: the monotone and boolean ones. We have showed that some properties are analogous as in the work of Popescu for free creation operators. However, especially for the monotone case, which we base on the weakly monotone creation operators, our results differ from the free case.

There is still a lot about the noncommutative joint numerical and spectral radii to be understood. Some open problems appeared in our study, in particular to find the exact formulas for the joint monotone numerical radius of $d \geq 2$ weakly monotone annihilation operators (at first glance it seems to be related to $\sqrt{d}$ rather then to $d$ ). But to see the real power of these objects one should search for analogues of the classical power inequality or von Neumann type inequalities. Finally, it would be interesting to know what are the relations between the three types (free, boolean and monotone) of numerical radii. 
Acknowledgements AK was supported by the Polish National Science Center Grant SONATA 2016/21/ D/ST1/03010. JW was supported by the Polish National Science Center Grant OPUS 2016/21/B/ST1/ 00628. AK and JW were also supported by the Polish National Agency for Academic Exchange (NAWA) within the POLONIUM program PPN/BIL/2018/1/00197/U/00021.

\section{Compliance with ethical standards}

Conflict of interest The authors declare that they have no conflict of interest.

Open Access This article is licensed under a Creative Commons Attribution 4.0 International License, which permits use, sharing, adaptation, distribution and reproduction in any medium or format, as long as you give appropriate credit to the original author(s) and the source, provide a link to the Creative Commons licence, and indicate if changes were made. The images or other third party material in this article are included in the article's Creative Commons licence, unless indicated otherwise in a credit line to the material. If material is not included in the article's Creative Commons licence and your intended use is not permitted by statutory regulation or exceeds the permitted use, you will need to obtain permission directly from the copyright holder. To view a copy of this licence, visit http:// creativecommons.org/licenses/by/4.0/.

\section{References}

1. Berger, C.A., Stampfli, J.G.: Mapping theorems for the numerical range. Amer. J. Math. 89, $1047-1055$ (1967)

2. Bożejko, M.: Positive definite functions on the free group and the noncommutative Riesz product. Boll. Un. Mat. Ital. 5(1), 13-21 (1986)

3. Bożejko, M.: Deformed Fock spaces, Hecke operators and monotone Fock space of Muraki. Demonstr. Math. 45(2), 399-413 (2012)

4. Crismale, V., Griseta, M.E., Wysoczański, J.: Weakly monotone Fock space and monotone convolution of the Wigner Law. J. Theor. Probab. 33, 268-294 (2020)

5. Goldberg, M., Tadmor, E.: On the numerical radius and its applications. Linear Algebra Appl. 42, 263-284 (1982)

6. Gustafson, Karl E., Rao, D.K.M.: Numerical range. The field of values of linear operators and matrices. Springer, New York (1997)

7. Muraki, N.: A new example of non-commutative "de Moivre-Laplace theorem". In: S. Watanabe et al (eds.), Prob. Th. Math. Sat., 7th Japan-Russia Symp., World Scientific, Tokyo, 1995, pp. 353-362 (1996)

8. Muraki, N.: Monotonic independence, monotonic central limit theorem and monotonic law of small numbers. Infin. Dimens. Anal. Quantum Probab. Relat. Top. 4(1), 39-58 (2001)

9. Popescu, G.: Unitary invariants in multivariable operator theory. Mem. Am. Math. Soc. 200, 941 (2009)

10. Toeplitz, O.: Das algebraische Analogon zu einem Satze von Fejar. Math. Z. 2(1-2), 187-197 (1918)

11. Voiculescu, D.: Symmetries of some reduced free product $C^{*}$-algebras, In: Operator algebras and their connections with topology and ergodic theory (Busteni, 1983), Lecture Note in Math., Vol. 1132 , pp.115-144. Springer, Heidelberg (1985)

12. Wysoczański, J.: Monotonic independence on the weakly monotone Fock space and related Poisson type theorem. Infin. Dimens. Anal. Quantum Probab. Relat. Top. 8(2), 259-275 (2005)

13. Wysoczański, J.: Monotonic independence associated with partially ordered sets. Inf. Dim. Anal. Quantum Probab. Relat. Top. 10(1), 17-41 (2007) 